\title{
Research on Portfolio Model Based on Multi-Objective Genetic Algorithm
}

\author{
Haonan $\operatorname{Lin}^{1, \text { a }}$ \\ ${ }^{1}$ South China University of Technology, Guangzhou, Guangdong, China, 510006 \\ aemail,
}

\begin{abstract}
Keywords: Portfolio, High Performance Computing, Multi-Objective Evolutionary Algorithm,
\end{abstract} Multiple Attribute Decision Making

\begin{abstract}
Portfolio optimization problem is a multi-objective optimization problem, it is necessary to consider the benefits should also consider the risks and the optimal situation is to achieve the least risk and maximum return. Papers define portfolio optimization problems, analyze the shortcomings of the existing portfolio model and the corresponding solution ideas, introduce multi-objective genetic algorithm and demonstrate the feasibility of multi-objective genetic algorithm in the application portfolio. Algorithm design process, detailed process of constructing and building a portfolio investment in line with the actual situation, put forward five objective optimization model from all angles, through a reasonable, optimized simulation test, and then select the algorithm selected from a number of programs better overall performance of the program.
\end{abstract}

\section{Introduction}

Internet banking, or financial technology, which can be divided into several areas of Direct Internet banking, Internet brokerage with a single social insurance field long tail wind control product design and marketing, and funds in the field of fund supermarket, small loans fast user portrait and wind control lending, consumer finance big data risk control, as well as P2P and crowd funding, the last is the robot's investment adviser, asset management, and portfolio.

Financial technology, the computer technology and how to combine with the financial industry are quite different in various areas, including in the portfolio and smart investment adviser, the core is one of the asset management algorithms.

Money management algorithm, or algorithms portfolio, is an investor in an uncertain environment for financial assets, the optimal choice and rational allocation decision problem from the perspective of data fields as well as scenarios of points, can be divided into the underlying assets ( stocks, futures, bonds, options, etc.) and asset allocation asset allocation fund category, the fund class data, performance data refer to the Fund's investment in the underlying assets after multiple product models or fund of funds management configuration.

From 1952 Markowitz mean variance model began to establish modern investment theory, based on the configuration data, people in the random case static mode a lot of research. Bell Labs Kelly, proposed optimal growth path problem, began to study the dynamic investment portfolio. The probability of investors in the stock market is difficult to predict the distribution of securities gains, return on assets showed fuzzy uncertainty and raised the possibility of the theory in 1978 in recent years scholars pay more attention to the fuzzy portfolio theory try to combine expertise, market liquidity, investor satisfaction and other factors. Since the 1980s, the financial "visions" have been found and interpreted, behavioral finance has been greatly developed, portfolio theory based on behavioral finance is undoubtedly a new research direction and has practical significance.

Application of artificial intelligence algorithms portfolio, also multi-party research, such as various classification algorithm, evolutionary algorithm, clustering algorithm, deep learning algorithms, can be applied to the portfolio, as well as multi-agent decision algorithm in which the application is also a relatively new research direction.

Most of the above asset allocation strategy to Markowitz mean - variance model, the goal is under the conditions of a given level of portfolio risk, the right to seek the highest expected return 
reconfiguration. However, this theory has a significant deficiency is that only consider the overall portfolio risk, while ignoring pose a risk. Through this approach to build a portfolio, there will often be a risk asset full control of the phenomenon, which is contrary to the concept of diversified investment.

In most of the above-mentioned algorithm research, largely based on the underlying asset data that is used to configure the algorithm portfolio asset base and optimize the static bias within the sample, but in practical application scenario, the second application scenario it is also very popular, and for a fund products, plays a vital role in the financial markets and the increasing complexity of change accelerates, risk identification and timely portfolio adjustments need now, but this is more a lack of study abroad.

\section{The Research Status}

The securities market is an extremely complex uncertainty system, the overall uncertainty can be divided into random uncertainty and fuzzy uncertainty

The Random Uncertainty Portfolio Model. Harry Markowitz published a paper "Portfolio Selection", which laid the foundation of modern portfolio theory, "mean-variance" theory provides a feasible method for the quantitative weigh the risks and benefits, the best investment in the securities market issues groundbreaking research. Sharpe in 1963 proposed a single exponential model, using a diagonal pattern simplifies the computational complexity of the original model. In terms of improved risk measurement, Mao and Swalm proposed the use of semi-variance method to measure the investment risk, the establishment of a mean - semi-variance model.

Kane, Kanno and Suzuki, Chunhachinda and so increases the skewness of this goal, the establishment of a mean - variance - skewness model. Roy proposed safety first model in 1952, as the basis for the theory of VAR.

Mossin proposed dynamic programming method, marking the portfolio theory from a single stage of research into multi-period. In 2000, Li and $\mathrm{Ng}$ with embedded multi-stage method to convert into a mean-variance problem resolvable problem, dynamic programming algorithm efficient frontier.

The Fuzzy Uncertainty Portfolio Model. Traditional portfolio selection is generally based on the uncertainty on, ignoring the ambiguity. Bellman proposed a fuzzy decision

Policy model, and for multi-objective decision-making systems, research portfolio model under fuzzy environment can be divided into three modules, Portfolio Selection Based on Fuzzy Decision, based on interval planning portfolio selection, based on the possibility of portfolio selection theory.

Since 2001, domestic and foreign scholars have conducted in-depth research, Tong in the target coefficients and coefficients are constrained interval number of cases.

The objective function is obtained worth range. Lai and other investment income and investment risk will be considered as interval numbers, raised three model portfolios. Zhang and put forward effective allowable deviation portfolio model and studied the clear possibility of variance and covariance fuzzy numbers.

Online Portfolio Model. 1991, Cover for the first time proposed a pre-funding allocation algorithm, so that the growth rate of close to afterwards (all prices given) optimal resource allocation algorithm (BCRP). The UP algorithm also opens the door to online portfolio selection research.

From the perspective of machine learning techniques, according to research into the work of the three aspects of the comb

The first category, "Follow-the-Winner", will increase earnings in the past preferred securities holdings, reducing earnings in the past with poor security, that momentum (inertia) trading strategy, which stems from future securities prices remain inertia hypothesis ; the main representatives of the UP Cover algorithm and Helmbold like EG algorithm; the second category, "Follow-the-Loser", assuming that the future price of securities is reversed, thus, will reduce earnings in the past better securities, revenue increased in the past with poor security, that reverse trading strategy; Bordin and $\mathrm{Li}$ and others launched online reverse investment strategy is no longer limited to looking for the 
best post-BCRP policy as an evaluation criterion pan-portfolio strategy, but to find and easy to calculate optimal or near-optimal online strategy according to the price volatility of the market, such a strategy is not beautiful and perfect analytical expressions of mathematical theory, but in a large number of experiments made cumulative gain significantly better than EG UP strategy and policy, and is conducive to large-scale computing. The third category, based on pattern recognition strategy, trying to use historical data to predict future prices of securities are subject to distribution and optimization model.

\section{The Innovation Point of the Research}

Financial issues have large scale, complexity, enormous and the results of high precision. For some complex financial issues, studies and research from practical application operability from the perspective of theoretical analysis, there is a big difference between the two is, and the method of theoretical analysis is often unsuitable for real-world applications. In recent years, with the rapid development of computer technology, financial sector emerged in an actual use of computers to solve the financial problems of the research boom. This will finance joint research and computer into the forefront of scientific research and financial applications. Now, based on the theoretical analysis, computer technology and the method of testing, it has become a major way today's financial research.

China's securities market has a huge group of individual investors. Now in the fund financial metropolis it is very popular. The reason most investors choose funds, because it is "professional financial management, risk diversification, risk-sharing." Expected rate of return and risk are people to invest most concerned about two issues. Usually when they want certain risk, the bigger the better earnings; or when certain benefits, risk, the smaller the better. However, investors are able to withstand a range of different risks are not the same. So based on the benefits and risks of multi-objective optimization genetic algorithm, we obtained the Pareto frontier, enrich the range of options investors to help them find the most satisfied with the investment program.

\section{Research Content}

The Definition of Multi-Objective Problem. Definition 1 multi-objective optimization problem $\min F(x)=\left(f_{1}(x), f_{2}(x), f_{3}(x), \ldots, f_{m}(x)\right) T$,

s.t.g $(x) \leqslant \mathrm{O}(\mathrm{i}=1,2, \ldots, \mathrm{P})$;

$\mathrm{h}(\mathrm{x})=\mathrm{O}(\mathrm{j}=1,2, \ldots, \mathrm{q}),(1)$

Where: $m$ is the number of objective function: $f_{i}, g_{i}, h_{j}: R^{n} \rightarrow R, x \in R^{n}$ decision variable; $X=\{x \mid$ $\left.\mathrm{x} \in \mathrm{R}^{\mathrm{n}}, \mathrm{g}_{\mathrm{i}}(\mathrm{x}) \leqslant \mathrm{O}, \mathrm{h}_{\mathrm{j}}(\mathrm{x})=\mathrm{O}, \mathrm{i}=1,2, \ldots, \mathrm{P} ; \mathrm{j}=1,2, \ldots, \mathrm{q}\right\}$ is called multi-objective optimization problem is feasible region.

Dominance relationship defined 2Pareto

Suppose $\mathrm{u}=\left(\mathrm{u}_{1}, \mathrm{u}_{2}, \ldots, \mathrm{um}\right) \mathrm{T}, \mathrm{v}=\left(\mathrm{v}_{1}, \mathrm{v}_{2}, \ldots, \mathrm{v}_{\mathrm{m}}\right) \mathrm{T} \in \mathrm{R}^{\mathrm{m}}$ to two target vector, if for all $\mathrm{i} \in$ $\{1,2, \ldots, \mathrm{m}\}$ such that $\mathrm{u}_{\mathrm{i}} \leqslant \mathrm{v}_{\mathrm{i}}$ and $\mathrm{u} \neq \mathrm{v}$, then uPareto dominated $\mathrm{v}$.

Define 3 Pareto solution set and Pareto frontier

If the decision space a point $\mathrm{x} * \in \mathrm{Rn}$, there is no other point $\mathrm{x}$ such that $\mathrm{F}(\mathrm{x})$ dominate $\mathrm{F}(\mathrm{x} *)$, then $\mathrm{x} *$ is called the global Pareto optimal solution. Optimal solution set consisting of all global called the Pareto set. For Pareto target vector $\mathrm{PF}=\{\mathrm{F}(\mathrm{x}) \in \mathrm{Rm} \mid \mathrm{x} \in \mathrm{PS}\}$ called Pareto frontier.

MOEA / D Algorithm. Optimization algorithm (MOEA / D) based on multi-objective decomposition is a new multi-objective optimization framework, the framework combines the advantages of local search and a number of multi-objective single-objective Pareto sampling. MOEA / D algorithm uses the Chebyshev method to a multi-objective optimization problem (MOP) into a plurality of sub-single-objective optimization problem.

Definition 4 Chebyshev method

Suppose $\mathrm{m}$ is the number of objects; $\lambda=\left(\lambda_{1}, \lambda_{2}, \ldots, \lambda_{\mathrm{m}}\right)$ is the weight vector satisfying $\Sigma \mathrm{m}_{\mathrm{i}}=1 \lambda_{\mathrm{i}}$ $=1 ; \Omega$ multi-objective optimization solution space and $\mathrm{z}^{*}=\left(\mathrm{z}_{1} *, \mathrm{z}_{2} *, \ldots, \mathrm{z}_{\mathrm{m}}{ }^{*}\right)$ as a reference 
point, the Chebyshev method can be expressed as $\min \mathrm{g}\left(\mathrm{x} \mid \lambda, \mathrm{z}^{*}=\max \left\{\lambda_{\mathrm{i}}\left|\mathrm{Fi}(\mathrm{x})-\mathrm{z}_{\mathrm{i}} *\right|\right\}\right.$;

s.t.x $\in \Omega$

In a multi-objective optimization $\mathrm{z}$ * shall comply with the additional constraint that $\mathrm{z}_{\mathrm{i}} *=\min \left\{\mathrm{F}_{\mathrm{i}}(\mathrm{x}) \mid \mathrm{x} \in \Omega\right\}$.

While using evolutionary algorithms for solving these single-objective optimization problem, to close the entire Pareto front of the goal. Algorithm to maintain a current optimal solution consisting of each sub-population problem, the definition of neighbor relations between sub-problems is a problem right from the child's weight vectors, each sub-optimization problems between neighboring sub-problems by evolutionary operation carries out. Succeeded in mathematical programming decomposition method commonly used in the algorithm is introduced to the evolution of the target areas, the distribution and diversity of fitness solving single objective optimization problem of retention policies directly applied to the MOEA / D algorithm.

Research Methods and Technical Route. Zhang et al in 2007, a multi-objective mathematical decomposition based on evolutionary algorithm (MOEA / D), the algorithm in solving multi-objective optimization problems exhibit efficiency beyond MOGLS (Multiple-objective genetic local search) algorithm, NSGA -II algorithm. Therefore, this article will be the first multi-objective optimization algorithm MOEA Chebyshev Decomposition / D applied to solving problems in the portfolio

Model Propulsion Optimization Mechanism. The market is evolving and over a period of time to optimize to get the law might be effective in the next period of time, but few long-term effective. If the law fails to update the model performance may not be satisfactory. Therefore, we need to continue to use the latest information in optimization, dynamic optimization of this law. Use of back testing and training set rolling stage can be a good combination to avoid failure problem.

In the actual experiment, we need to constantly adjust the portfolio, continuously optimized in order to continue to maintain effectiveness.

MOEA / D Algorithm Flow. Use Tchebycheff decomposition strategy of MOEA / D algorithm:

First, you need to save each generation the following information:

(1)Groups having $n$ points, $x^{1}, \ldots, x^{n}$, where $x i$ represents the current solution $i$-th issue;

(2) $F V^{1}, \ldots, F^{n} F V^{i}$ where $x i$ is the value of the objective function, i.e., for each $i=1, \ldots$, n has $\mathrm{FV}^{\mathrm{i}}=\mathrm{F}\left(\mathrm{x}^{\mathrm{i}}\right)$;

(3) $\mathrm{z}=\left(\mathrm{z}_{1}, \ldots \mathrm{z}_{\mathrm{m}}\right)$ where $\mathrm{z}_{\mathrm{i}}$ is the best value of the objective function $\mathrm{f}_{\mathrm{i}}$ currently found.

(4) a set of external group (Externalpopulation, EP). It used to hold non-dominated solutions found during the search.

Enter:

(1) multi-objective problem MOP;

(2) the termination condition

(3) n: number of sub-issues MOEA / D decomposition;

(4) $n$ a uniform distribution of the weight vector:, ...,

(5) T: Right in the neighborhood of the number of vectors.

Output: EP

Step 1 Initialization

1.1) so that $\mathrm{EP}=$

1.2) calculate the Euclidean distance between each of the two weight vectors and vector for each weight to their nearest $\mathrm{T}$ a weight vector composition of its neighbor. I.e., for each $\mathrm{i}=1, \ldots, \mathrm{n}$, there are $\mathrm{B}(\mathrm{i})=\left\{\mathrm{i}_{1}, \ldots \mathrm{i}_{\mathrm{T}}\right\}$,

Wherein, ..., $\mathrm{T}$ is the weight vector of a neighborhood.

1.3) randomly generating an initial population $x^{1}, \ldots, x^{n}$ and calculates $F V^{i}=F\left(x^{i}\right)$.

1.4) set the initial value $\mathrm{z}=\left(\mathrm{z}_{1}\right.$ according to specific issues, ..., $\left.\mathrm{zm}\right) \mathrm{T}$

Step 2 Update

For each $\mathrm{i}=1, \ldots, \mathrm{n}$, do the following

2.1) Reproduction: randomly select two numbers $k$ and $\mathrm{l}$ from $\mathrm{B}$ (i), and then for $\mathrm{x}^{\mathrm{k}}$ and $\mathrm{x}^{\mathrm{l}}$ 
genetic

2.2) improve the new solution, based on the specific issue of the solution is adjusted / repaired by y becomes y '.

2.3) Update $\mathrm{z}$ : for $\mathrm{j}=1, \ldots, \mathrm{m}$, if $\mathrm{z}_{\mathrm{j}}<\mathrm{f}_{\mathrm{j}}(\mathrm{y})$, then let $\mathrm{z}_{\mathrm{j}}=\mathrm{f}_{\mathrm{j}}(\mathrm{y})$.

2.4) update neighborhood Solution: For each individual numbers, if there is

Then let $\mathrm{x}=\mathrm{y}, \mathrm{FV}_{\mathrm{j}}=\mathrm{F}(\mathrm{y})$.

2.5) Update EP: EP delete all the target vector $F$ is dominated, if not dominated, then EP in any target vector, which was added in the EP.

Step 3 termination conditions

If function evaluations than or equal to Gmax, stop counting and outputs the EP. Otherwise, return to step 2.

MOEA / D by the decomposition of sub-problems and each individual one to one population that reduce computational complexity, to accelerate the convergence speed groups.

\section{Experimental Test}

The use of small experiments show the feasibility of using multi-objective genetic algorithm in the portfolio in question, the experimental data for the risky asset returns 14 curves in Figure 3, with a total capital of 10 million, the test data from April 2010 No. 16-2015 February 1.

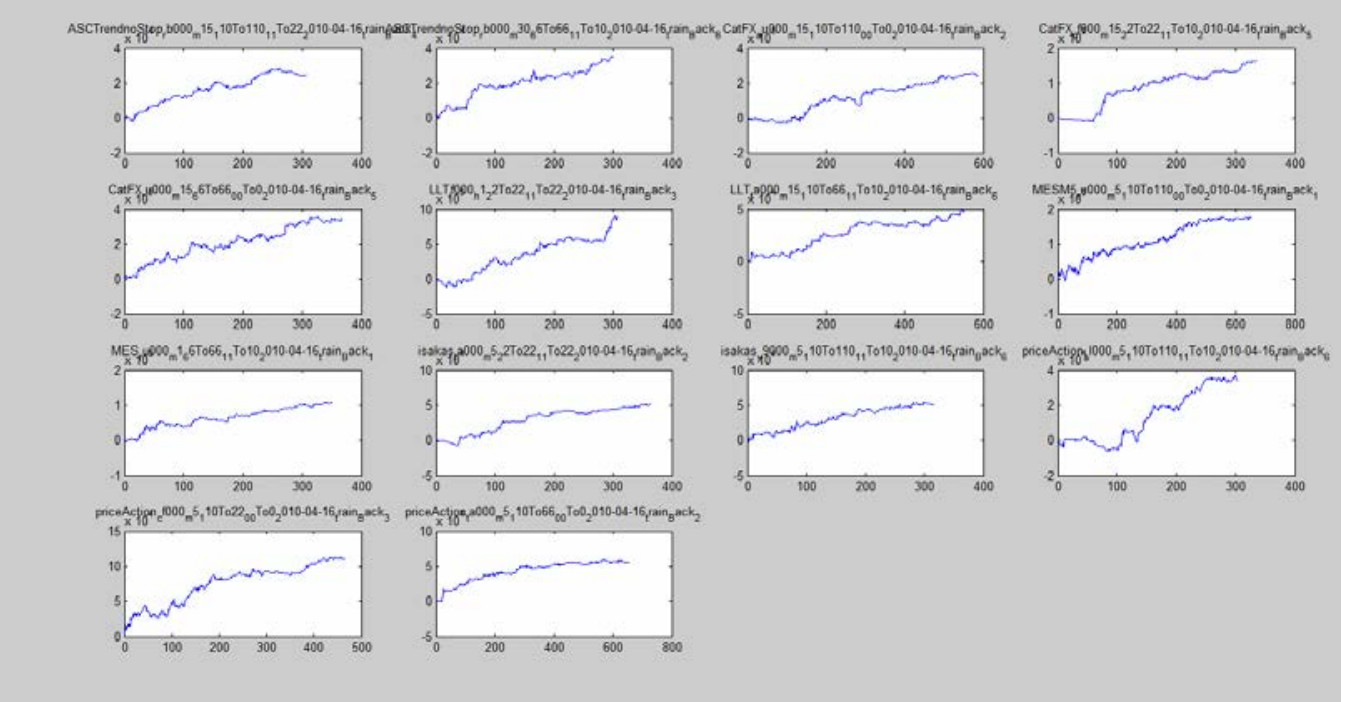

Figure 1 Test Sample

Parameter MOEA / D is the number of the population to take 600, the number of neighbors to take 100, 200 take Algebra, select the training data and test data, in days, training days were $30,60,90,180$ days, the number of days for test 30,60,90,180 days, combined two by two, in a few days of training more than the number of days equal to the test under the circumstances, a plurality of sets of training test, were tested, and finally selected the best ratio.

Benchmark test results for the average distribution of funds for each sequence, the model effective or not, is whether the target value is better than in most benchmarks.

Finally screened through portfolio optimization algorithm target sequence compared with the average distribution of the reference sequence shown in Table 1 and Figure 2.

Table 1. Comparison of experimental results

\begin{tabular}{|c|c|c|c|c|c|c|}
\hline & $\begin{array}{c}\text { Rate of } \\
\text { Return }\end{array}$ & $\begin{array}{c}\text { Standard } \\
\text { Deviation }\end{array}$ & $\begin{array}{c}\text { The Maximum } \\
\text { Retracement } \\
\text { Value }\end{array}$ & $\begin{array}{c}\text { The Maximum } \\
\text { Retracement } \\
\text { Rate }\end{array}$ & Winning & Odds \\
\hline $\begin{array}{c}\text { Reference } \\
\text { Sequence }\end{array}$ & 17.9697 & 0.0844 & $3.4697 * 10^{7}$ & 0.2629 & 0.4080 & 1.1387 \\
\hline $\begin{array}{c}\text { Sequence } \\
\text { Optimization }\end{array}$ & 13.2840 & 0.0238 & $5.5707 * 10^{6}$ & 0.1360 & 0.4295 & 1.7981 \\
\hline
\end{tabular}




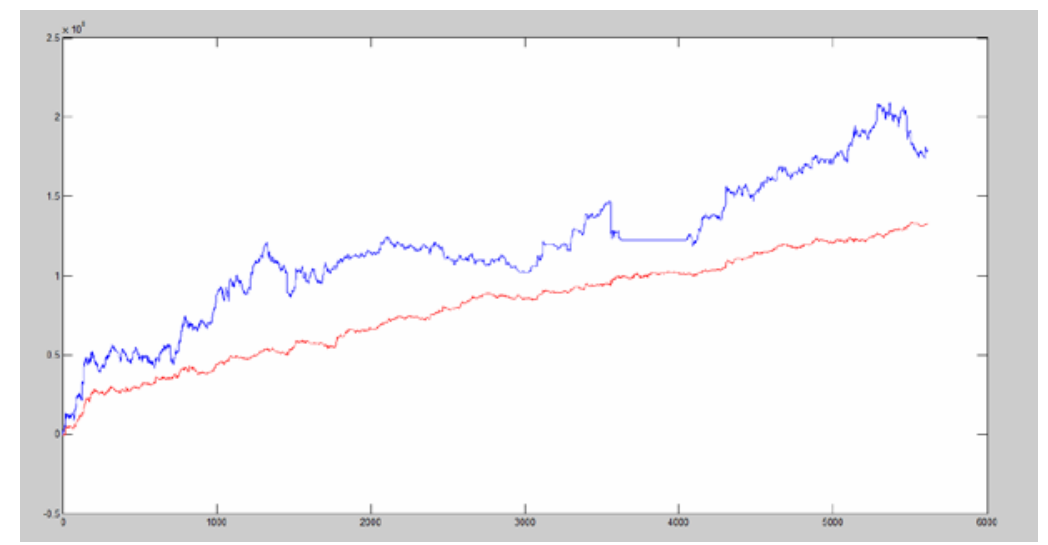

Figure 2 Comparison of experimental results

Seen from the results in the standard deviation, the maximum retracement, maximum retracement rate risk indicators point of view, the effect of a clear asset portfolio algorithm, fully reflects the risk aversion functions, from the perspective of winning and odds, it can also be seen only after a asset allocation algorithm optimization, steady growth in assets significantly strengthened ability, more in line with actual investment needs.

Experimentally proved by the initial multi-objective genetic algorithm in the portfolio has a certain practical significance and research value.

\section{Conclusion}

Resource allocation optimization problem is a practical and have unlimited exploration of possible problems, whether in the computer field, or in the financial and economic fields, there are countless people to explore. Direction of asset management in the financial sector of the Internet, we need for our customers to develop a reasonable program of personalized portfolio. Asset management combining algorithm can be abstracted into a multi-objective optimization process, it is necessary to consider the benefits should also consider the risks, or even to consider transaction costs and capital utilization. The main use of artificial intelligence algorithms, a plurality of operations research theory, game theory, behavioral finance theory, fuzzy theory systemic solution to this problem, define the portfolio optimization problem, new scenarios, based on investment in recent years in practice problems encountered, such as the actual transaction amount of data, model-sample failure, unreasonable risk measure, into multiple sub-topics, namely research, research models proposed in the framework of cooperation in selected before, form a more stereo system.

\section{References}

[1] Li, B., Zhao, P., Hoi, S.C.H. and Gopalkrishnan, V. Machine Learning, Vol. 2 (2012) No 87, p.77-91

[2] Borodin, A., El-Yaniv, R. and Gogan, V. Journal of Artificial Intelligence Research, Vol. 12 (2005) No 27, p.74-76

[3] Helmbold, D.P, Singer, Y., Schapir, R.E., et al. Insurance: Mathematics and Economics, Vol. 30 (2014) No 19, p.144-145

[4] Zhang, W., Zhang, X., and Chen, Y. Insurance: Mathematics and Economics, Vol. 29 (2012) No 27, p.21-23

[5] Zhang, X., Zhang W. and Cai, R. Journal of Computational and Applied Mathematics, Vol. 8 (2013) No 27, p.57-60 